\title{
Modelo matemático Two-echelon Capacitated Vehicle Routing Problem para a logística de distribuição de encomendas
}

\author{
Karina Pedrini Fraga ${ }^{1}$, Rodrigo de Alvarenga Rosa ${ }^{2}$, Glaydston Mattos Ribeiro ${ }^{3}$, Carlos Antonio Silva ${ }^{4}$ e \\ Raimundo Franca Júnior ${ }^{4}$
}

\begin{abstract}
Resumo: Muitas cidades vêm enfrentando dificuldades de mobilidade urbana e, por isso estão impondo restrições para à circulação de caminhões de maior porte. Assim, empresas de logística vêm atuando em uma logística de dois níveis usando Centros de Distribuição Urbanos (CDU) que recebem caminhões de maior porte e fracionam a carga para distribuir aos clientes em caminhões de pequeno porte. Para colaborar com este planejamento, este artigo realiza uma adaptação de um modelo matemático baseado no Two-echelon Capacitated Vehicle Routing Problem (2E-CVRP) para resolver de forma integrada a formação de rotas entre o depósito central e os CDU e as rotas destes para os clientes. O modelo foi aplicado à logística dos Correios na região metropolitana do Espírito Santo e instâncias com até 4 CDU e 25 clientes foram testadas com o uso do solver CPLEX 12.6 obtendo as rotas para as entregas nos dois níveis.
\end{abstract}

Palavras-chave: Two-Echelon Capacitated Vehicle Routing Problem, frota heterogênea, restrição de acesso logística reversa.

\begin{abstract}
Many cities are facing difficulties in urban mobility and therefore are imposing restrictions on the movement of larger trucks. Thus, logistics companies developed a two level logistics strategy based on Urban Distribution Centers (CDU) that receives larger trucks and split the cargo to put in small trucks to distribute to customers. To support this type of logistics planning, this paper presents an adaptation of a mathematical model based on the Two-echelon capacitated Vehicle Routing Problem (2E-CVRP) to plan the routes from the central depot to the satelites and from these to the clients. The model was applied to the logistics of Correios in the metropolitan area of the Espírito Santo, Brazil, and instances with up to 4 CDU and 25 clients were tested using CPLEX solver 12.6 obtaining routes for deliveries at both levels.
\end{abstract}

Keywords: Two-Echelon Capacitated Vehicle Routing Problem, heterogeneous fleet, site dependence, reverse logistic.

\section{INTRODUÇÃO}

A mobilidade urbana vem enfrentando problemas que têm motivado a imposição de restrições legais a veículos de grande porte e com cargas pesadas em mais de 100 cidades brasileiras (Portal Transporta Brasil, 2014). Essas restrições visam minimizar os impactos como o congestionamento e a poluição sonora e do ar por meio da diminuição ou supressão da circulação de veículos de grande porte em vias urbanas, o que pode resultar na melhoria da qualidade de vida da população. Uma possível opção de reduzir a circulação de veículos de grande porte nas cidades é estruturar a logística de distribuição de cargas utilizando Centros de Distribuição Urbana (CDU). Os CDU são instalações localizadas preferencialmente próximas à entrada das cidades ou junto a terminais aéreos, ferroviários ou portuários e destinam-se a fracionar cargas recebidas por meio de veículos de grande porte em veículo menores, que fazem a distribuição dentro do perímetro urbano (Crainic et al., 2009; Soysal et al., 2014).

Após a revisão da literatura, pode-se constatar que o modelo que mais se adequa à realização do roteamento de veículos no contexto da logística urbana por meio do uso de

\footnotetext{
${ }^{1}$ Karina Pedrini Fraga, Mestrado em Engenharia Civil - Transportes,

UFES. (kpfraga@yahoo.com.br)

${ }^{2}$ Rodrigo de Alvarenga Rosa, Mestrado em Engenharia Civil -

Transportes, UFES. (rodrigo.a.rosa@ufes.br)

${ }^{3}$ Glaydston Mattos Ribeiro, Programa de Engenharia de Transportes,

COPPE-UFRJ. (glaydston@pet.coppe.ufrj.br)

${ }^{4}$ Carlos Antonio Silva, CORREIOS/SA. (casilva@correrios.com.br)

${ }^{5}$ Raimundo Franca Júnior, CORREIOS/SA. (rfjunior@correios.com.br)
}

Manuscrito recebido em 16/03/2016 e aprovado para publicação em $25 / 08 / 2016$

Este artigo é parte de TRANSPORTES v. 24, n. 3, 2016. ISSN: 2237-1346 (online). DOI:10.4237/transportes.v24i3.1095
CDU é o Two-echelon Capacitated Vehicle Routing Problem (2E-CVRP). O 2E-CVRP foi proposto recentemente e embora possua poucas publicações na área, tem recebido cada vez mais atenção em periódicos com alto fator de impacto (Jepsen; Ropke e Spoorendonk, 2013).

Assim, este artigo visa adaptar o modelo matemático 2E-CVRP proposto por Perboli et al. (2011) para a logística de distribuição de cargas em 2 níveis, com o uso de CDU. $\mathrm{O}$ modelo difere do modelo proposto por Perboli et al. (2011) por sua função objetivo. No modelo proposto neste artigo, a função objetivo além de minimizar os custos de transporte e de carregamento e descarregamento, como proposto por Perboli et al. (2011), busca minimizar também o tamanho da frota do primeiro e do segundo níveis. Essa mudança na função objetivo é importante, pois várias empresas operam com frotas alugadas e, usualmente, nesta situação, o custo do aluguel do veículo é muito superior ao custo do quilômetro rodado. Assim, com essa mudança, é possível avaliar o impacto do custo da frota no custo total de distribuição.

O modelo proposto foi aplicado ao planejamento da entrega de encomendas dos Correios na Região Metropolitana da Grande Vitória, Espírito Santo. A distribuição de encomendas pelos Correios na Região Metropolitana da Grande Vitória (RMGV) tem início no Centro de Tratamento de Cartas e Encomendas (CTCE), para onde são encaminhadas todas as cargas destinadas a clientes dessa região. Do CTCE, as encomendas são enviadas aos Centros de Entregas e Encomendas (CEE), de acordo com a distância do cliente a ser atendido. Atualmente, existem cinco CEE na RMGV: um na cidade de Vitória, um na cidade de Vila Velha, um em Cariacica, um em Viana e um em Serra. 


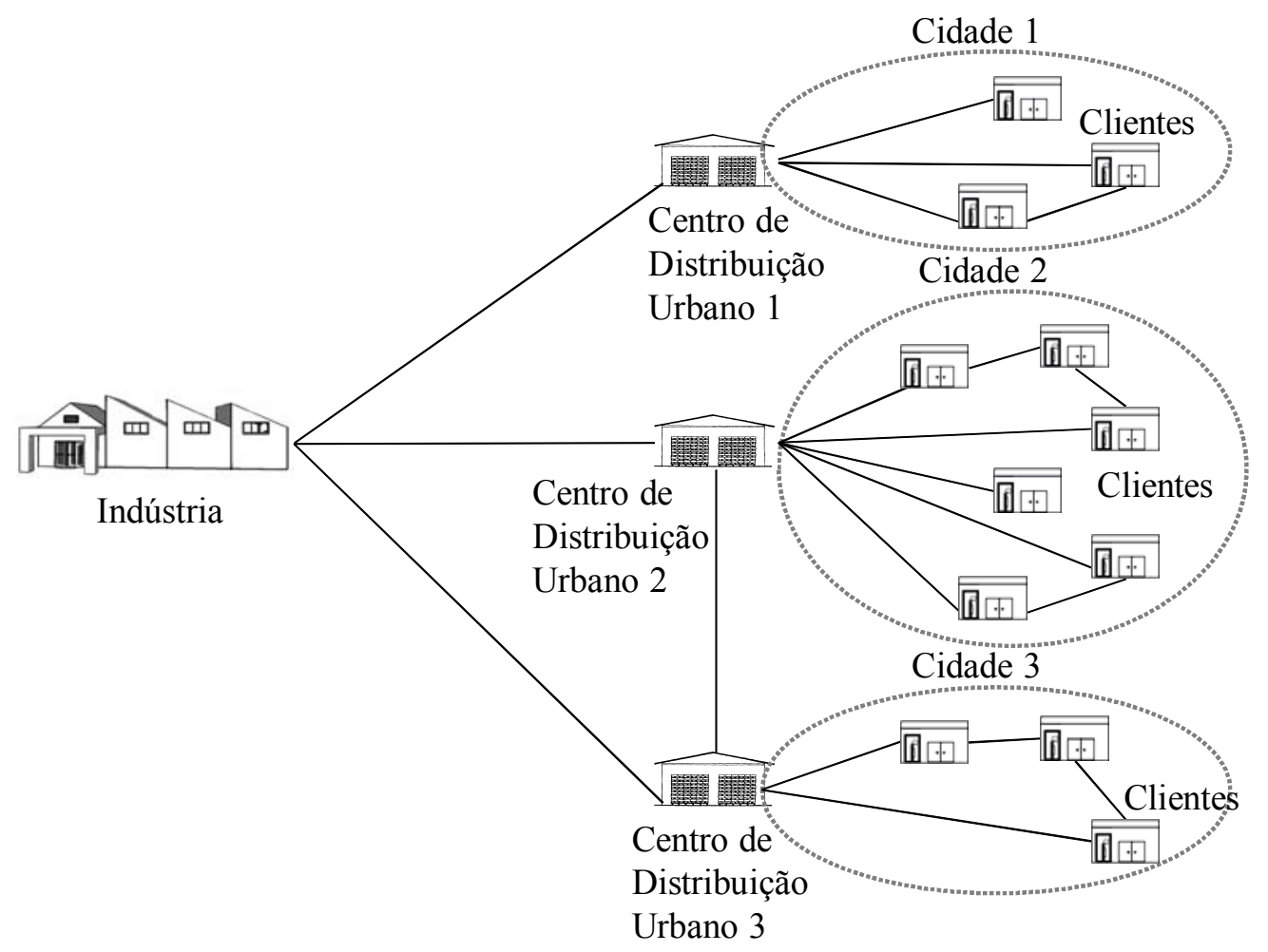

Figura 1. Centro de Distribuição Urbano

Fonte: Adaptado de Quak (2008, p.67)

Do CEE, as cargas são enviadas a pontos estratégicos, num total de 25 pontos, de onde são entregues aos destinatários. Este artigo se propõe a resolver o planejamento da distribuição de cargas do CTCE até os 25 pontos estratégicos, não abrangendo o destinatário final, visto que para atender esse elo da cadeia é utilizada uma outra frota para a realização do transporte. Assim, a cadeia de distribuição de encomendas dos Correios pode ser modelada em dois níveis, sendo o primeiro nível composto pelo transporte do CTCE para os CEE e o segundo nível composto pelo transporte dos CEE para os pontos estratégicos.

$\mathrm{O}$ restante deste artigo está organizado da seguinte forma: a Seção 2 apresenta conceitos sobre o 2E-CVRP, assim como uma revisão da literatura acerca do 2E-CVRP. A Seção 3 detalha o modelo matemático proposto neste artigo. A Seção 4 apresenta o o problema real estudado e descreve as instâncias analisadas na aplicação do 2E-CVRP. Os resultados do modelo proposto e suas análises são apresentados na Seção 5. Por fim, as conclusões são apresentadas na Seção 6.

\section{REFERENCIAL TEÓRICO}

Tendo em vista as novas opções logísticas de dividir a rede de distribuição ou coleta em vários níveis, é possível obter, além da redução dos custos de transportes, ganhos no ponto de vista ambiental, já que com a distribuição em vários níveis é viável a utilização de veículos menores para a realização de entregas a curtas distâncias, o que reduz a poluição do ar e o congestionamento das vias urbanas (Mancini, 2013).

Na maioria das vezes, a logística é implementada em dois níveis e para tal são implantados Centros de Distribuição Urbana (CDU) (Crainic et al., 2009). Os CDU são instalações localizadas preferencialmente próximas à entrada das cidades ou junto a transportes aéreos, ferroviários ou terminais de navegação. Os CDUs destinam-se a fracionar cargas recebidas por meio de veículos de grande porte em veículo menores, que fazem a distribuição dentro do perímetro urbano (Crainic et al., 2009; Mancini, 2013). Podese ver na Figura 1 um desenho esquemático da distribuição de cargas por meio de um CDU.

Para desenvolver um roteamento de veículos que suporte a logística urbana por meio do uso de CDU, o problema de roteamento em dois níveis que tem sido mais citado na literatura é o Two-echelon Capacitated Vehicle Routing Problem (2E-CVRP) (Cuda et al., 2015). O 2ECVRP é um problema NP-hard de distribuição em dois níveis, que analisa o problema em dois níveis, sendo composto por depósitos e satélites, que são depósitos intermediários que funcionam como CDU, fracionando as cargas recebidas em veículos menores para serem entregues aos clientes. No primeiro nível, é realizado o transporte das cargas demandadas pelos clientes do depósito aos satélites e, no segundo nível, a entrega dos satélites aos clientes. Nos satélites ocorrem atividades de descarregamento dos veículos do primeiro nível e carregamento dos veículos do segundo nível (Baldacci et al., 2013; Perboli e Tadei, 2010; Soysal et al., 2014).

O 2E-CVRP tem por objetivo encontrar um conjunto de rotas para cada nível, de maneira a satisfazer a demanda de todos os clientes, respeitando as capacidades dos satélites e dos veículos, com a minimização do custo total de distribuição. Embora o transporte em sistemas de distribuição de dois níveis só permita entregas em um mesmo nível, o problema não deve ser resolvido tratando cada nível separadamente. Isto se deve ao fato que eventualmente a otimização da rota do primeiro nível que define as quantidades entregues aos satélites não leve obrigatoriamente à rotas ótimas no segundo nível, pois a escolha dos volumes entregues 
aos satélites pode não permitir a reelização de rotas ótimas no segundo nível (Cuda; Guastaroba; Speranza, 2015).

Como os veículos do segundo nível possuem capacidade menor que a capacidade dos veículos do primeiro nível, o 2E-CVRP é de grande relevância para o atendimento de clientes localizados em cidades que possuem regulamentações que restrinjam o tráfego de grandes veículos em suas vias, bastando a inserção de satélites em tais cidades (Baldacci et al., 2013; Cuda et al., 2015; Soysal et al., 2014).

Perboli et al. (2011) apresentaram formalmente a classe Two-echelon Vehicle Routing Problem e sua variação mais básica, 2E-CVRP, assim como um modelo de Programação Inteira Mista e Inequações para a resolução deste problema, apresentando contribuições ao trabalho de Feliu et al. (2007). Perboli et al. (2011) propuseram também duas matheuristics, dividing-based heuristic e semicontinuous heuristic e utilizaram instâncias encontradas na literatura acerca do CVRP para testar o modelo e as heurísticas.

Jepsen et al. (2013) propuseram uma heurística Branch-and-cut para o 2E-CVRP. Sitek e Wikarek (2014) desenvolveram uma nova abordagem para a modelagem e resolução do 2E-CVRP ao integrar modelos matemáticos PLIM com Programação Lógica por Restrições (PLR). Santos et al. (2013) desenvolveram uma reformulação da Programação Inteira para o 2E-CVRP e implementaram duas heurísticas Branch-and-price (BP) para a sua solução.

Crainic et al. (2008) propuseram as heurísticas Splitlarge-route, Add and Exchange para o problema 2E-CVRP, baseadas nas abordagens de clustering e multi depósitos. Crainic et al. (2010) resolveram o 2E-CVRP com o uso de uma família de heurísticas Multi-Start, de maneira que cada nível foi tratado com um subproblema e resolvido separadamente e sequencialmente, num processo iterativo. Um algoritmo híbrido Ant Colony Optimization foi desenvolvido por Meihua et al. (2011) a partir da combinação de três meta-heurísticas para a resolução do $2 \mathrm{E}-\mathrm{CVRP}$. O método resolve o problema dividindo-o em subproblemas CVRPs e as metaheurísticas Ant Colony Optimization e Multiple Neighborhood Descent são combinadas para a resolução desses CVRP. Em seguida, a meta-heurística ThresholdBased Local Search é utilizada para melhorar a solução viável encontrada anteriormente. Hemmelmayr et al. (2012) resolveram o 2E-CVRP no contexto da Logística Urbana com o desenvolvimento de uma meta-heurística Adaptive Large Neighborhood Search (ALNS).

Crainic et al. (2012) solucionaram o 2E-CVRP com o uso de meta-heurísticas baseadas em Greedy Randomized Adaptive Search Procedure (GRASP) combinado com Path Relinking. Baldacci et al. (2013) desenvolveram um modelo matemático com base em programação dinâmica (PD), em um método dual-ascent e um algoritmo exato para resolver o 2E-CVRP. Zeng et al. (2014) desenvolveram uma heurística híbrida, composta de GRASP e de uma Variable Neighborhood Descent (VND) para resolução e melhoria da solução, respectivamente, do 2E-CVRP.

Como artigos de revisão, podem ser encontrados os trabalhos de Mancini (2013) e Cuda et al. (2015). Mancini (2013) revisou os problemas de roteamento existentes na literatura de Logística Urbana envolvendo multi-echelon distribution systems (ME-DS), inclusive o 2E-CVRP e os métodos utilizados para sua resolução. Cuda et al. (2015) fizeram um estudo das publicações sobre Two-echelon Routing Problems, em que abordaram oito principais artigos da classe 2E-CVRP.
Variações do 2E-CVRP também são encontradas na literatura. Crainic et al. (2009) apresentaram uma variação do 2E-CVRP, chamada de two-echelon, synchronized, scheduled, multi-depot, multiple-tour, heterogeneous vehicle routing problem with time windows (2SS-MDMTVRPTW), ao tratar o gerenciamento da Logística Urbana. Esses autores desenvolveram um modelo e formulações gerais para a nova classe a partir de Programação Linear Inteira, mas não realizaram nenhum experimento computacional para a mesma. Grangier et al. (2014) abordaram uma nova classe do 2E-CVRP, chamada two-echelon multipletrip vehicle routing problem with sattelite synchronization (2E-MTVRP-SS) e utilizaram uma meta-heurística Adaptive Large Neighborhood Search para resolução do problema. Soysal et al. (2014) abordaram pela primeira vez a variação time-dependent em problemas 2E-CVRP, o Twoechelon Capacitated Vehicle Routing Problem with Time Dependent (2E-CVRPTD), assim como fatores que influenciam no consumo de combustível, como o tipo de veículo, a distância percorrida, a velocidade e a carga transportada pelo veículo. Esses autores desenvolveram um modelo matemético de PLIM baseada no modelo proposto por Jepsen et al. (2013) e testaram o modelo em um caso real, uma cadeia de suprimentos localizada nos Países Baixos, com 1 depósito, 2 satélites e 16 clientes.

\section{MODELO MATEMÁTICO PROPOSTO}

O modelo matemático foi adaptado do modelo matemático proposto por Perboli et al. (2011), modificando a função objetivo que passa a considerar o custo da frota do primeiro nível e do segundo nível. De acordo com Perboli et al. (2011), o 2E-CVRP é composto por um depósito e por um número conhecido de satélites. Cada satélite possui localização conhecida e sua capacidade é definida como a quantidade máxima de veículos do segundo nível que podem iniciar uma rota a partir desse satélite. Cada nível possui uma frota homogênea de veículos, sendo que a frota de veículos do primeiro nível encontra-se localizada no depósito e a frota de veículos do segundo nível é compartilhada pelos satélites.

No primeiro nível, as rotas possuem início e fim no depósito, enquanto no segundo nível as rotas devem terminar no satélite de origem. Cada veículo do primeiro nível pode transportar produtos de um ou mais clientes e atender mais de um satélite por rota, mas não é permitido que sejam realizadas entregas diretamente do depósito aos clientes. Cada satélite pode ser servido por um ou mais veículos do primeiro nível, enquanto cada cliente só pode ser atendido por meio de um único veículo do segundo nível. Assim, considera-se que entregas fracionadas só podem ocorrer no primeiro nível. O custo total de distribuição é dado pelos custos das rotas realizadas nos dois níveis e pelo custo de manuseio das cargas nos satélites, sendo este custo proporcional à carga carregada/descarregada.

A diferença entre o modelo proposto e o modelo de Perboli et al. (2011) consiste na mudança do Função Objetivo do modelo. Enquanto o modelo de Perboli et al. (2011) tem uma Função Objetivo que busca minimizar o custo das viagens realizadas e o custo de manuseio das cargas, a Função Objetivo do modelo proposto visa minimizar o custo das viagens realizadas, o custo do manuseio das cargas e o tamanho da frota de cada nível. 
O 2E-CVRP pode ser definido como um grafo não orientado $G=(N, E)$, onde o conjunto de vértices $N$ é dado como $N=V_{0} \cup V_{s} \cup V_{c} . \mathrm{N}=\mathrm{V}_{0} \cup \mathrm{V}_{\mathrm{s}} \cup \mathrm{V}_{\mathrm{c}}$. $\mathrm{O}$ vértice $V_{0}=\left\{v_{0}\right\}$ representa o depósito, enquanto $V_{s}=\left\{V_{s_{1}}, V_{s_{2}}, \ldots, V_{s_{n s}}\right\}$ representa 0 subconjunto dos $n_{s}$ satélites e $V_{c}=\left\{n_{s}+1, n_{s}+2, \ldots, n_{s}+n_{c}\right\}$ o subconjunto dos $n_{c}$ clientes. $\mathrm{O}$ conjunto das arestas $E$ é definido como $E=\left\{\{0, j\}: j \in V_{s}\right\} \cup\left\{(i, j): i, j \in\left\{V_{s} \cup V_{c}\right\}, i<j\right\}$ e representa as arestas que interligam os vértices.

O modelo é composto pelos parâmetros:

$m_{1}$ : número de veículos do primeiro nível;

$m_{2}$ : número de veículos do segundo nível;

$m_{s_{k}}$ : número máximo de rotas do segundo nível que

podem se iniciar em um satélite $k$;

$K^{1}$ : capacidade dos veículos do primeiro nível;

$K^{2}$ : capacidade dos veículos do segundo nível;

$d_{i}$ : demanda de um cliente $i$

$c_{i j}$ : custo de percorrer um $\operatorname{arco}(i, j)$;

$F_{k}$ : custo de operação por $\mathrm{m}^{3}$ de carga manuseada no depósito intermediário $k$;

$f^{1}$ : custo do veículo do primeiro nível; e

$f^{2}$ : custo do veículo do segundo nível.

As variáveis de decisão são divididas em três grupos. No primeiro grupo são definidos dois conjuntos de variáveis de decisão relacionadas aos arcos, um para cada nível:

$x_{i j}$ : representa a quantidade de veículos do primeiro nível que percorrem um arco $(i, j)$ do primeiro nível; e

$y_{i j}^{k}$ : variáveis binárias que assumem valor igual a $1 \mathrm{se}$ um veículo do segundo nível sai de um nó $i$ e chega a um nó $j$, ambos do segundo nível, em uma rota iniciada em um satélite $k$. Caso contrário, seu valor é 0 . decisão:

O segundo grupo contém as seguintes variáveis de

$z_{k j}$ : variáveis binárias que assumem valor igual a 1 quando um cliente $j$ deve ser servido por um satélite $k$ e valor igual a 0 em caso contrário. Essa variável é responsável por relacionar cada cliente a um depósito e por ligar os dois níveis de transporte.

O terceiro grupo representa o fluxo de carga que passa através de cada arco e de cada satélite:

$Q_{i j}^{1}$ : indica o fluxo de carga que passa em um arco $(i, j)$ do primeiro nível;

$Q_{i j k}^{2}$ : indica o fluxo de carga que passa por um arco $(i, j)$ do segundo nível, em uma rota iniciada em um satélite $k$; e, por fim, télite $k$.

$D_{k}$ : define a quantidade de carga que passa pelo sa-

Função objetivo
Minimizar

$$
\begin{aligned}
& \sum_{i, j \in V_{0} \cup V_{S}, i \neq j} c_{i j} x_{i j}+\sum_{k \in V_{S}} \sum_{i, j \in V_{S} \cup V_{C}, i \neq j} c_{i j} y_{i j}^{k}+\sum_{k \in V_{S}} F_{k} D_{k}+ \\
& \sum_{i \in V_{S}} x_{0 i} f_{1}+\sum_{k \in V_{S}} \sum_{j \in V_{C}} y_{k j}^{k} f_{2}
\end{aligned}
$$

Sujeito a:

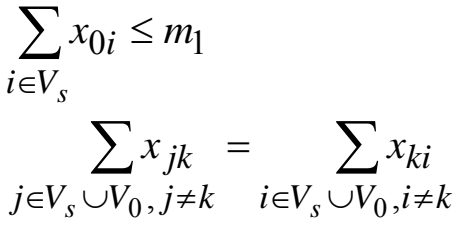

$$
\begin{aligned}
& \sum_{k \in V_{s}} \sum_{j \in V_{c}} y_{k j}^{k} \leq m_{2} \\
& \sum_{j \in V_{c}} y_{k j}^{k} \leq m_{s_{k}}
\end{aligned}
$$$$
\forall k \in V_{S} \cup V_{0}
$$

$\forall k \in V_{S}$

$$
\sum_{j \in V_{c}} y_{k j}^{k}=\sum_{j \in V_{c}} y_{j k}^{k}
$$

$\forall k \in V_{S}$

$\sum_{i \in V_{s} \cup v_{0}, i \neq j} Q_{i j}^{1}-\sum_{i \in V_{s} \cup v_{0}, i \neq j} Q_{j i}^{1}=\left\{\sum_{i \in V_{c}}^{D_{j}}-d_{i}\right.$

$$
\forall j \in V_{S} \cup V_{0}
$$

$$
Q_{i j}^{1} \leq K^{1} x_{i j}
$$

$$
\begin{gathered}
\sum_{i \in V_{C} \cup k, i \neq j} Q_{i j k}^{2}-\sum_{i \in V_{C} \cup k, i \neq j} Q_{j i k}^{2}= \\
\begin{cases}z_{k j} d_{j} & j \text { não é um satélite } \\
-D_{j} & j \text { é um satélite }\end{cases}
\end{gathered}
$$

$Q_{i j k}^{2} \leq K^{2} y_{i j}^{k}$

$$
\forall j \in V_{C} \cup V_{S}, \forall k \in V_{S}
$$

$\sum_{i \in V_{s}} Q_{i v_{0}}^{1}=0$

$\forall i, j \in V_{C} \cup V_{S}, i \neq j, \forall k \in V_{S}$

$$
\sum_{j \in V_{c}} Q_{j k k}^{2}=0
$$




$$
D_{k}=\sum_{j \in V_{c}} d_{j} z_{k j}
$$

$\forall k \in V_{S}$

$$
y_{i j}^{k} \leq z_{k j} \quad \forall i \in V_{s} \cup V_{c}, \forall j \in V_{c}, \forall k \in V_{s}
$$

$$
y_{j i}^{k} \leq z_{k j}
$$

$$
\sum_{i \in V_{s} \cup V_{c}} y_{i j}^{k}=z_{k j}
$$

$$
\forall i \in V_{s}, \forall j \in V_{c}, \forall k \in V_{S}
$$

$$
\sum_{i \in V_{s}} y_{j i}^{k}=z_{k j}
$$

$$
\forall j \in V_{C}, \forall k \in V_{S}
$$

$$
\sum_{i \in V_{s}} z_{i j}=1
$$

$$
\forall j \in V_{C}, \forall k \in V_{S}
$$

$$
\forall j \in V_{C}
$$

$$
y_{k j}^{k} \leq \sum_{l \in V_{s} \cup V_{0}} x_{k l}
$$

$$
\forall j \in V_{C}, \forall k \in V_{S}
$$

$y_{i j}^{k} \in\{0,1\}$

$$
z_{k j} \in\{0,1\}
$$

$$
\forall i, j \in V_{C}, \forall k \in V_{s} \cup V_{0}
$$

$$
\forall j \in V_{C}, \forall k \in V_{S} \cup V_{0}
$$

$x_{i j} \in \mathrm{Z}^{+}$

$$
\forall k, j \in V_{S} \cup V_{0}
$$

$Q_{i j}^{1} \geq 0$

$$
\forall i, j \in V_{s} \cup V_{0}
$$

$$
Q_{i j k}^{2} \geq 0
$$

$$
\forall i, j \in V_{S} \cup V_{C}, \forall k \in V_{S}
$$

Depois das definições expostas anteriormente, são apresentadas a seguir a função objetivo e as restrições do modelo proposto.

O modelo possui uma função objetivo, representada pela Equação (1), que é composta por cinco parcelas. A $1^{\text {a }}$ e a $2^{\mathrm{a}}$ parcelas consistem nos somatórios dos custos das viagens realizadas através dos arcos do primeiro e do segundo níveis, respectivamente.

A $3^{\mathrm{a}}$ parcela representa o custo de carregamento e descarregamento das cargas em cada satélite. A $4^{\mathrm{a}}$ e $5^{\mathrm{a}}$ parcelas apresentam os somatórios dos custos das frotas de primeiro e segundo níveis, respectivamente. A função objetivo deve ser minimizada.
A Restrição (2) determina que o número de rotas percorridas no primeiro nível não pode exceder a quantidade de veículos da frota de primeiro nível. Da mesma maneira, na Restrição (4), o número de rotas de segundo nível não pode exceder o número de veículos da frota de segundo nível. As Restrições (3) indicam que, para $k=0$, ou seja, se $k$ for o depósito, cada rota do primeiro nível deve iniciar e terminar no depósito. Se $k$ for um satélite, essas restrições garantem que o número de veículos que chegam ao satélite é igual ao número de veículos que saem desse mesmo satélite.

As Restrições (5) garantem que o número de rotas que iniciam em um satélite $k$ não pode exceder o número máximo de rotas que podem ser iniciadas nesse satélite. As Restrições (6) definem que toda rota de segundo nível que se iniciar em um determinado satélite $k$ deve retornar ao mesmo satélite $k$. De acordo com as Restrições (7) e (9), o fluxo resultante de carga em cada nó, no primeiro e no segundo nível, respectivamente, deve ser igual à demanda desse nó, com exceção do depósito, onde o fluxo resultante deve ser igual à carga total demandada pelos clientes, com sinal negativo.

As Restrições (8) asseguram que o fluxo passando num $\operatorname{arco}(i, j)$ do primeiro nível não deve exceder a capacidade dos veículos de primeiro nível utilizados nesse arco. Nas Restrições (10), o fluxo passando num $\operatorname{arco}(i, j)$ do segundo nível não deve exceder a capacidade dos veículos do segundo nível utilizados nesse arco. As Restrições (11) e (12) garantem que, em cada rota do primeiro nível e do segundo nível, não retorna carga para o depósito e para o satélite onde a rota se iniciou, respectivamente.

As Restrições (13) definem a quantidade de carga que passa pelo satélite $k$. As Restrições (14) e (15) garantem que um cliente é servido por um satélite somente se receber carga advinda desse satélite. As Restrições (16) e (17) garantem que apenas uma rota do segundo nível passa em cada cliente, ou seja, cada cliente é visitado apenas uma vez. As Restrições (18) asseguram que cada cliente é atendido por apenas um satélite. As Restrições (19) estabelecem que uma rota do segundo nível se inicia em um satélite $k$ somente se esse satélite já foi atendido por uma rota do primeiro nível. As Restrições (20) a (24) determinam o domínio das variáveis, sendo $y_{i j}^{k}$ e $z_{k j}$ variáveis binárias, $x_{i j}$ variáveis de valor inteiro e maior que zero, e $Q_{i j k}^{2}$ variáveis maiores ou iguais à zero.

\section{DESCRIÇÃO DO PROBLEMA E LEVANTAMENTO DOS DADOS}

Foram realizadas diversas reuniões com a equipe de distribuição de encomendas dos Correios, eminentemente caixas Sedex, para entender a logística atual de distribuição. Além disso, por meio dessas reuniões foi possível determinar os parâmetros necessários para a resolução do problema. Com isso, a cadeia de distribuição de encomendas dos Correios foi adaptada ao sistema de distribuição twoechelon considerando o CTCE como o depósito; os CEE como os satélites; e os pontos estratégicos como os clientes. Dessa forma, o primeiro nível é composto pelo CTCE e pelos CEE e o segundo nível é composto pelos CEE e os clientes. Um esboço desse sistema é ilustrado na Figura 2. 


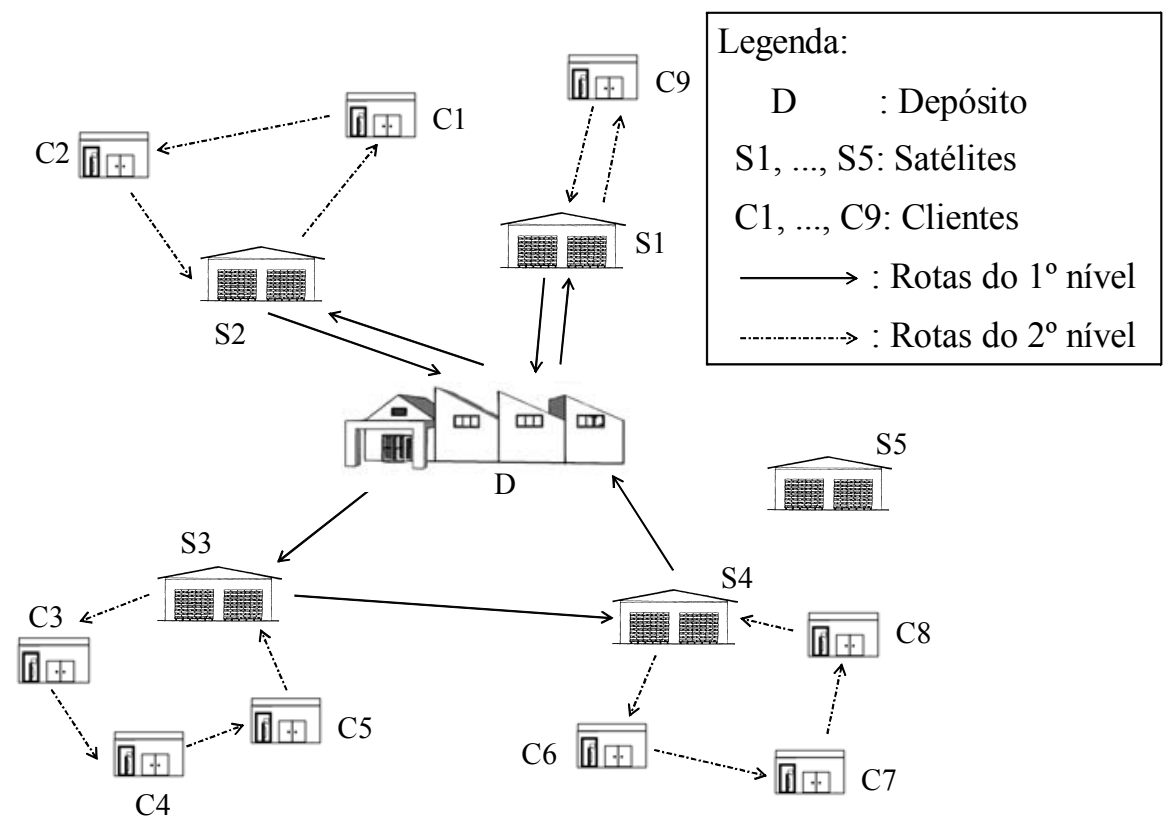

Figura 1. Distribuição modal dos acessos e das viagens atraídas ao CAA

Foram criados três grupos de instâncias de teste: $01^{\circ}$ grupo, Instâncias 1 a 3 , com 10 clientes a serem atendidos em cada instância; o $2^{\circ}$ grupo, Instâncias 4 a 6 , com 15 clientes a serem atendidos em cada instância; e o $3^{\circ}$ grupo, Instâncias 7 a 9, com 25 clientes a serem atendidos em cada instância. Foram definidas nove instâncias de teste de maneira a possibilitar a análise do comportamento do modelo com a variação do número de clientes a serem atendidos e dos satélites disponíveis para a realização do fracionamento da carga. Dentro de cada grupo de instâncias de teste variou-se o número de satélites e manteve-se constante o número de clientes a serem atendidos.

Além disso, foi criado o $4^{\circ}$ grupo, com a Instância 10 que, assim como a Instância 9, retrata o problema real, em que existem 4 satélites que atendem 25 clientes com a diferença que na Instância 10, os 4 satélites obrigatoriamente têm que ser utilizados. A Tabela 1 apresenta todas as instâncias.

Na Tabela 1, a coluna Grupo representa os grupos de instâncias de teste. A coluna Instância representa todas instâncias reais e de teste definidas e utilizadas. A coluna Satélites indica a quantidade de satélites em cada instância e a coluna Cliente apresenta a quantidade de clientes considerados em cada instância. As colunas Veículos 1 e Veículos 2 representam, respectivamente, o número de veículos do primeiro e do segundo níveis.
Tabela 1. Instâncias Avaliadas

\begin{tabular}{cccccc}
\hline Grupo & Instância & $\begin{array}{c}\text { Satélites } \\
\text { (un) }\end{array}$ & $\begin{array}{c}\text { Clientes } \\
\text { (un) }\end{array}$ & $\begin{array}{c}\text { Veículos 1 } \\
\text { (un) }\end{array}$ & $\begin{array}{c}\text { Veículos 2 } \\
\text { (un) }\end{array}$ \\
\hline \multirow{3}{*}{1} & 1 & 2 & 10 & 2 & 4 \\
& 2 & 3 & 10 & 2 & 4 \\
& 3 & 4 & 10 & 2 & 4 \\
\hline \multirow{3}{*}{2} & 4 & 2 & 15 & 3 & 8 \\
& 5 & 3 & 15 & 3 & 8 \\
& 6 & 4 & 15 & 3 & 8 \\
\hline \multirow{3}{*}{3} & 7 & 2 & 25 & 3 & 8 \\
& 8 & 3 & 25 & 3 & 8 \\
\hline 4 & 9 & 4 & 25 & 3 & 8 \\
\hline
\end{tabular}

A quantidade de cargas entregue via Correios foi definida a partir da proporção de cartas entregue a cada cliente diariamente. De acordo com os Correios, a proporção de encomendas entregues aos clientes tem correlação com a quantidade de cargas entregues.

As distâncias entre todos os nós envolvidos no problema foram determinadas a partir do cálculo da distância entre CEP, realizado com o auxílio do site MapaCep (2015). Os custos de transporte para cada nível foram estimados com preço de mercado em $\mathrm{R} \$ 2,00 / \mathrm{km}$ percorrido no primeiro nível e em $\mathrm{R} \$ 1,50 / \mathrm{km}$ percorrido no segundo nível. O custo de manuseio é o mesmo para todos os satélites, visto que este custo é dado por licitação e deve ser padronizado para todas as unidades e foi considerado como $\mathrm{R} \$ 1,00$ por $\mathrm{m}^{3}$ de encomendas manuseadas nos satélites.

Tabela 2. Resultados Encontrados pelo CPLEX

\begin{tabular}{|c|c|c|c|c|c|c|c|}
\hline Grupo & Instância & $\begin{array}{c}\text { Satélites } \\
\text { disponíveis }\end{array}$ & $\begin{array}{c}\text { Satélites } \\
\text { utilizados }\end{array}$ & $\begin{array}{c}\text { Clientes } \\
\text { (um) }\end{array}$ & $\begin{array}{c}\text { Veículos utilizados } \\
1 \text { (um) }\end{array}$ & $\begin{array}{c}\text { Veículos utilizados } \\
2 \text { (um) }\end{array}$ & $\begin{array}{c}\text { Função } \\
\text { Objetivo (R\$) }\end{array}$ \\
\hline \multirow{3}{*}{1} & 1 & $1 ; 2$ & $1 ; 2$ & 10 & 2 & 3 & $48.302,00$ \\
\hline & 2 & $1 ; 2 ; 3$ & $1 ; 2 ; 3$ & 10 & 2 & 3 & $48.322,00$ \\
\hline & 3 & $1 ; 2 ; 3 ; 4$ & $1 ; 2 ; 3$ & 10 & 2 & 3 & $48.343,00$ \\
\hline \multirow{3}{*}{2} & 4 & $1 ; 2$ & 2 & 15 & 1 & 3 & $41.282,00$ \\
\hline & 5 & $1 ; 2 ; 3$ & $3 ; 2$ & 15 & 1 & 3 & $41.289,00$ \\
\hline & 6 & $1 ; 2 ; 3 ; 4$ & $3 ; 2$ & 15 & 1 & 3 & $41.316,00$ \\
\hline \multirow{3}{*}{3} & 7 & $1 ; 2$ & $1 ; 2$ & 25 & 2 & 4 & $78.515,00$ \\
\hline & 8 & $1 ; 2 ; 3$ & $1 ; 2$ & 25 & 2 & 4 & $78.482,00$ \\
\hline & 9 & $1 ; 2 ; 3 ; 4$ & $1 ; 3 ; 4$ & 25 & 2 & 4 & $78.520,00$ \\
\hline 4 & 10 & $1 ; 2 ; 3 ; 4$ & $1 ; 2 ; 3 ; 4$ & 25 & 2 & 4 & $78.578,00$ \\
\hline
\end{tabular}




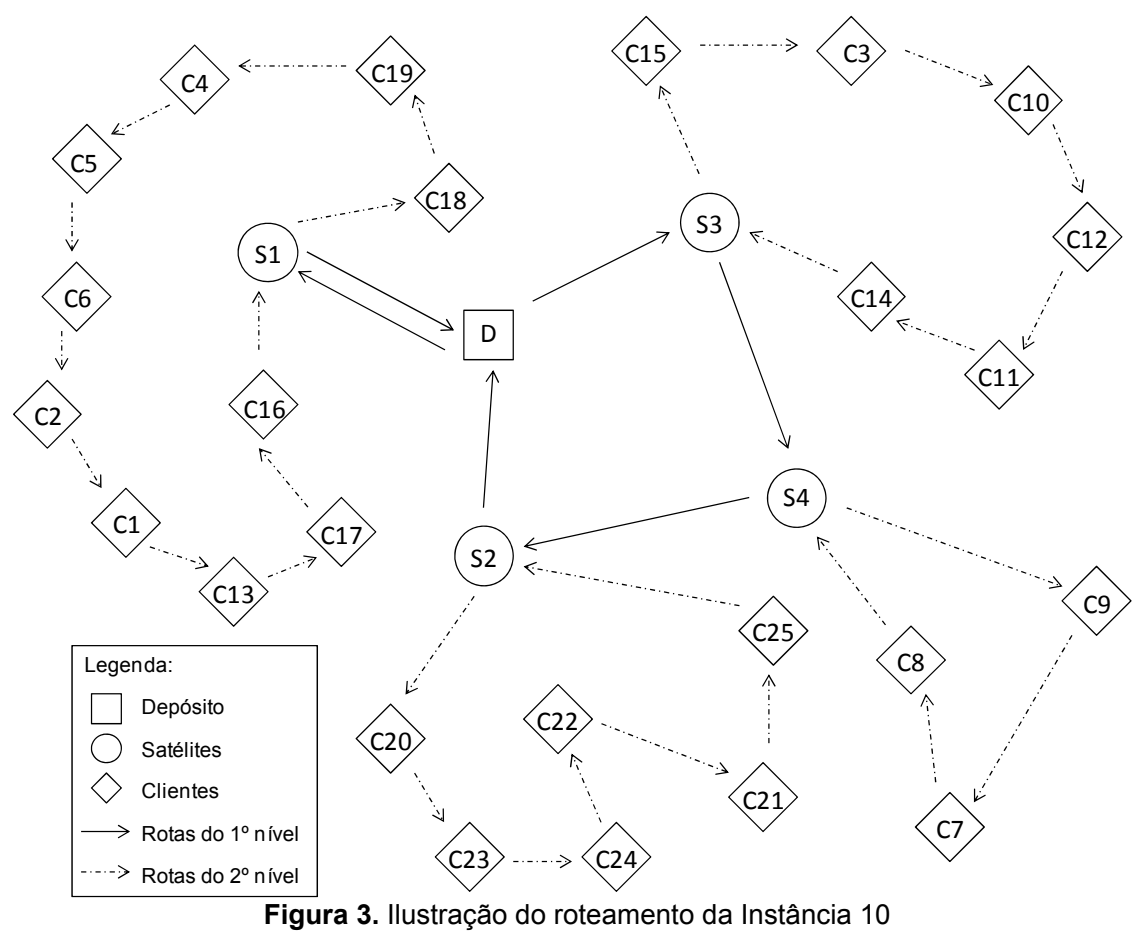

Os valores dos veículos do primeiro nível e do segundo nível são, respectivamente, $\mathrm{R} \$ 19.000,00$ e $\mathrm{R} \$ 10.000,00$.

Para a distribuição das encomendas do CTCE aos CEE (primeiro nível) é utilizada uma frota homogênea de veículos com capacidade maior que a frota homogênea de veículos utilizada para a entrega das encomendas dos CEE aos clientes (segundo nível). Os valores relativos à capacidade dos veículos e aos custos para obtê-los sofreram incrementos de acordo com a demanda determinada para cada instância, mantendo-se constantes dentro de cada grupo de instância, de maneira que os veículos das instâncias do Grupo 3 possuem capacidade maior que os veículos das instâncias do Grupo 1 e do Grupo 2; e os veículos das instâncias do Grupo 2 possuem capacidade maior que os veículos das instâncias do Grupo 1, independentemente da quantidade de satélites disponíveis para a instância. Para a definição da capacidade dos veículos, foi utilizado apenas $60 \%$ da capacidade total dos veículos do segundo nível em função da dificuldade de se arrumar as cargas no veículo, sobretudo, para respeitar a sequência de entrega na arrumação do caminhão.

\section{APRESENTAÇÃO E ANÁLISE DE RESULTADOS}

Os resultados foram alcançados após a execução das instâncias reais e de teste, definidas na Seção 4, com o uso do solver CPLEX 12.6. A Tabela 2 mostra o resumo desses resultados. Na Tabela 2, a primeira coluna indica o grupo a que cada instância pertence e a segunda coluna identifica cada instância. A coluna Satélites disponíveis exibe quais satélites estavam disponíveis para a realização da distribuição e a coluna Satélites utilizados apresenta quais satélites foram utilizados. A coluna Clientes indica a quantidade de clientes a serem atendidos em cada instância. As colunas Veículos utilizados 1 e Veículos utilizados 2 indicam a quantidade de veículos utilizados para a distribuição de encomendas no primeiro nível e no segundo nível, respectiva- mente. A coluna Função Objetivo apresenta o valor encontrado para a função objetivo com a implementação de cada instância.

O CPLEX resolveu as Instâncias de 1 a 8 e 10, respeitando as restrições de capacidade e de fluxo.

O CPLEX não encontrou a solução ótima para a Instância 9, chegando a um upper bound (UB) de 78.520,00, como apresentado na Tabela 2, e a um lower bound (LB) de $78.512,00$, com um gap de $0,1 \%$ após 28 horas de execução do CPLEX. No entanto, foi observado que, com 7 minutos de execução do CPLEX, o modelo já apresentava o mesmo valor de UB, tendo alcançado um LB de 69.203,00, ou seja, após praticamente 28 horas não houve melhora no UB, subindo muito lentamente o LB.

Os valores que mais impactaram na Função Objetivo foram os valores correspondentes ao custo de obtenção das frotas do primeiro e do segundo níveis. Isso porque o valor de obtenção da frota utilizada é de grandeza maior que os outros custos. Com isso, as variações na Função Objetivo podem ser explicadas, principalmente, pelo tamanho das frotas a serem utilizadas para realizar a distribuição das encomendas nos dois níveis. A quantidade de clientes a serem atendidos e os satélites utilizados também influenciam o valor da Função Objetivo, por determinarem o tamanho da rota a ser percorrida e, no caso dos clientes, a quantidade de encomendas a serem entregues, que são manuseadas nos satélites e geram um custo de manuseio.

De acordo com a Tabela 2 , nenhuma instância utilizou a capacidade total dos veículos disponíveis e as Instâncias de 3 a 9 não utilizaram todos os satélites disponíveis para cada instância apresentada na Tabela 1 no resultado do roteamento realizado pelo CPLEX. Inclusive, uma comparação entre as Instâncias reais 9 e 10 indica que a utilização de três satélites em vez dos quatro utilizados para o atendimento dos atuais 25 clientes gera uma economia no custo total de distribuição. Assim, para o problema estudado, uma análise por parte da gerência dos Correios deve ser realizada a fim de se avaliar a perspectiva de não utilizar o Satélite 2, levando-se em consideração o custo do armazém que pode ser economizado e o reflexo dessa alteração nos custos da 
empresa, assim como questões comerciais. A Figura 3 ilustra o roteamento obtido na resolução da Instância 10.

Diante do exposto, pode-se perceber que o modelo proposto é capaz de promover uma logística de distribuição integrada entre os dois níveis do problema da logística de distribuição de encomendas dos Correios, com a minimização dos custos de transporte e do tamanho da frota a ser utilizada e com a evidência dos satélites mais interessantes para a realização do roteamento, de acordo com a sua localização. Assim, o modelo proposto pode servir aos Correios como ferramenta para planejamento da logística de distribuição de encomendas, mas também ao planejamento estratégico da empresa.

\section{CONCLUSÕES}

Este artigo propôs uma adaptação de um modelo matemático para o problema de distribuição em dois níveis $2 \mathrm{E}-$ CVRP para a logística de distribuição de encomendas dos Correios estruturada em dois níveis. A adaptação do modelo teve como base o modelo matemático 2E-CVRP proposto por Perboli et al. (2011) e diferencia-se dele por considerar o custo da frota em cada nível na função objetivo.

O modelo é capaz de promover uma logística de distribuição integrada entre os dois níveis do problema, mostrando a frota necessária nos dois níveis para o atendimento de todos os clientes e os satélites mais interessantes para a realização do roteamento, não considerando, no entanto, os custos de se manter cada satélite, mas apenas a sua localização. Os resultados indicaram que é possível realizar a logística dos Correios reduzindo o número de satélites, gerando uma economia no custo total de distribuição.

Assim, para o problema estudado, uma análise por parte da gerência dos Correios deve ser realizada a fim de se avaliar a perspectiva de não utilizar um satélite, levandose em consideração o custo do armazém que pode ser economizado e o reflexo dessa alteração nos custos da empresa, assim como questões comerciais. Cabe aos gestores dos Correios fazerem uma análise da importância da existência dos satélites não escolhidos pelo modelo para a realização do fracionamento das cargas a serem entregues aos clientes. O CPLEX conseguiu resolver o modelo matemático de forma ótima em instâncias com até 28 nós e a instância com 29 nós resultou em um pequeno gap de $0,1 \%$.

A metodologia apresentada pode servir como uma ferramenta de logística para os Correios e para sistemas similares. No entanto, por se tratar de um problema NP-hard, o modelo proposto para o 2E-CVRP não resolveria problemas com instâncias muito maiores que as apresentadas. Assim, como sugestão para trabalhos futuros, sugere-se a criação de meta-heurísticas para resolução de problemas com

\section{AGRADECIMENTOS}

Os autores agradecem a CAPES, à FAPES (458/2013 e processo 75528452/2016) e ao CNPq (477148/2011-5 e $313408 / 2014-9$ ) pelo apoio financeiro à pesquisa e aos Correios pelo apoio às informações sobre o problema estudado.

\section{REFERÊNCIAS}

Baldacci, R.; Mingozzi, A. e Roberti, R. (2013) An Exact Algorithm for the Two-Echelon Capacitated Vehicle Routing Problems. Operations Research, v. 61, n. 2, p. 298-314. DOI: 10.1287/opre.1120.1153

Crainic, T.G.; Mancini, S.; Perboli, G. e Tadei, R. (2012) GRASP with path relinking for the two-echelon vehicle routing problem. CIRRELT, v. 45.

Crainic, T. G.; Mancini, S.; Perboli, G.; Tadei, R. (2008) Lower Bounds for the Two-Echelon Vehicle Routing Problem. Anais do EU/MEETING, Troyes, France.

Crainic, T.G.; Mancini, S.; Perboli, G. e Tadei, R. (2010) Multistart heuristics for the two-echelon vehicle routing problem. CIRRELT, v. 30.

Crainic, T. G.; Ricciardi, N. e Storchi, G. (2009) Models for Evaluating and Planning City Logistics Systems. CIRRELT, v. 11.

Cuda, R.; Guastaroba, G. e Speranza, M. G. (2015) A survey on two-echelon routing problems. Computers \& Operations Research, v. 55, p. 185-199. DOI: 10.1016/j.cor.2014.06.008

Feliu, J. G.; Perboli, G.; Tadei, R. e Vigo, D. (2007) The Twoechelon Capacitated Vehicle Routing Problem. Technical Report, Control and Computer Department Politecnico de Torino, University of Bologna, Bologna, Italy.

Grangier, P.; Gendreau, M.; Lehuédé, F. e Rousseau, L. M. (2014) An Adaptive Large Neighborhood Search for the TwoEchelon Multiple-Trip Vehicle Routing Problem with Satellite Synchronization. CIRRELT, v. 33.

Hemmelmayr, V. C.; Cordeau, J. F. e Crainic, T. G. (2012) An adaptive large neighborhood search heuristic for two-echelon vehicle routing problems arising in city logistics. Computers \& Operations Research, v. 39, p. 3215-28. DOI:

10.1016/j.cor.2012.04.007

Jepsen, M.; Røpke, S. e Spoorendonk, S. (2013) A branch-andcut algorithm for the symmetric two-echelon capacitated vehicle routing problem. Transportation Science., v. 47, n. 1, p. 23-37. DOI: $10.1287 /$ trsc. 1110.0399

Mancini, S. (2013) Multi-Echelon Distribution Systems in City Logistics. European Transport, v. 54, n. 2.

MAPACep. Pesquisa geral no site. Disponível em: $<$ http://www.mapacep.com.br/distancia-ceps.asp $>$. Acesso em: 04 jul. 2015.

Meihua, W.; Xuhong, T.; Shan, C. e Shumin, W. (2011) Hybrid ant colony optimization algorithm for two echelon vehicle routing problem. Procedia Engineering, v. 15, p. $3361-3365$. DOI: $10.1155 / 2014 / 517467$

Perboli, G. e Tadei, R. (2010) New Families of Valid Inequalities for the Two-Echelon Capacitated Vehicle Routing Problems. Electronic Notes in Discrete Mathematics, v. 36, p. 639-646. DOI:10.1016/j.endm.2010.05.081

Perboli, G.; Tadei, R. e Vigo, D. (2011) The Two-Echelon Capacitated Vehicle Routing Problems. Transportation Science, v. 45 , n. 3 , p. $364-380$. DOI: $10.1287 /$ trsc. 1110.0368 
Portal Transporta Brasil (2014). Pesquisa geral no site.

Disponível em:

$<$ http://www.transportabrasil.com.br/2014/11/mais-de-cem-

municipios-do-brasil-ja-criaram-restricao-ao-transito-de-

caminhoes/>. Acesso em: 03 jul. 2015.

Quak, H. (2008) Sustainability of Urban Freight Transport Retail Distribution and Local Regulations in Cities. Erasmus research institute of management, Rotterdam.

Santos, F. A.; Da Cunha, A. S. e Mateus, G. R. (2013) Branchand-price algorithms for the Two-Echelon Capacitated Vehicle Routing Problem. Optimization Letters, v. 7, p. 1537-1547. DOI: 10.1007/s11590-012-0568-3

Sitek, P. e Wikarek J. (2014) A novel integrated approach to the modelling and solving of the Two-Echelon Capacitated Vehicle Routing Problem. Production \& Manufacturing Research: An Open Access Journal, v. 2, n. 1, p. 326-340. DOI:

$10.1080 / 21693277.2014 .910716$

Soysal, M., Bloemhof-Ruwaard, J. M. e Bektas, T. (2014) The time-dependent two-echelon capacitated vehicle routing problem with environmental considerations. International Journal of

Production Economics, v. 164, p. 366-378. DOI:

10.1016/j.ijpe.2014.11.016

Zheng-Yang, Z.; Wei-Sheng, X.; Zhi-Yu, X.; Wei-Hui, S. (2014) A Hybrid GRASP+VND Heuristic for the Two-Echelon Vehicle

Routing Problem Arising in City Logistics. Mathematical

Problems in Engineering, v. 2014, p. 1-11. DOI:

$10.1155 / 2014 / 517467$ 